\title{
OPEN A glutamate synthase mutant of Bradyrhizobium sp. strain ORS285 is unable to induce nodules on Nod factor-independent Aeschynomene species
}

\author{
Nico Nouwen ${ }^{\bowtie}$, Clémence Chaintreuil, Joel Fardoux \& Eric Giraud
}

The Bradyrhizobium sp. strain ORS285 is able to establish a nitrogen-fixing symbiosis with both Nod factor (NF) dependent and NF-independent Aeschynomene species. Here, we have studied the growth characteristics and symbiotic interaction of a glutamate synthase (GOGAT; gltD::Tn5) mutant of Bradyrhizobium ORS285. We show that the ORS285 gltD::Tn5 mutant is unable to use ammonium, nitrate and many amino acids as nitrogen source for growth and is unable to fix nitrogen under freeliving conditions. Moreover, on several nitrogen sources, the growth rate of the gltB::Tn5 mutant was faster and/or the production of the carotenoid spirilloxanthin was much higher as compared to the wild-type strain. The absence of GOGAT activity has a drastic impact on the symbiotic interaction with NF-independent Aeschynomene species. With these species, inoculation with the ORS285 gltD::Tn5 mutant does not result in the formation of nodules. In contrast, the ORS285 gltD::Tn5 mutant is capable to induce nodules on NF-dependent Aeschynomene species, but these nodules were ineffective for nitrogen fixation. Interestingly, in NF-dependent and NF-independent Aeschynomene species inoculation with the ORS285 gltD::Tn 5 mutant results in browning of the plant tissue at the site of the infection suggesting that the mutant bacteria induce plant defence responses.

Rhizobia are able to establish a symbiotic interaction with leguminous plants. In this interaction, the rhizobium induces the plant to form a new organ, the nodule, infected by the bacteria which differentiate into dinitrogen fixing bacteroids. This symbiotic nitrogen fixation not only allows leguminous plant to grow on nitrogen poor soils but also has a high impact in natural environments as well as in agriculture as it is one of largest contributors of nitrogen input into the soil.

The Bradyrhizobium sp. strain ORS285 is a photosynthetic bacterium that forms a symbiotic interaction with tropical aquatic legumes of the Aeschynomene genus ${ }^{1}$. It infects plants at the base of lateral roots or the adventitious root primordia on the stem via colonization of intercellular spaces between the epidermis and outer cortex plant cells. After this initial intercellular infection, the bacteria penetrate some plant cells and these infected cortical cells start to divide rapidly and repeatedly to form the nodule primordium ${ }^{2}$. The ORS285-Aeschynomene interaction is a fascinating symbiotic model because, the ORS285 strain, differently to the other photosynthetic bradyrhizobia, such as ORS278 and BTAi1, has nod genes and is able to nodulate a broader host range of Aeschynomene species that differ by the requirement or not of Nod Factors (NFs) to trigger nodulation ${ }^{3}$.

In a large screen for mutations that affect the symbiosis between the photosynthetic Bradyrhizobium strain ORS278 and the NF-independent Aeschynomene species, Aeschynomene indica, strains with Tn5 insertions into the $g l t B$ or $g l t D$ gene had a Nodule development defective $\left(\mathrm{Ndv}^{-}\right)$phenotype ${ }^{4}$. The $g l t B$ and $g l t D$ genes encode for the large and small subunit of the glutamate synthase, also known as glutamine 2-oxoglutarate amidotransferase (GOGAT) complex, respectively (Fig. 1A) Together with glutamine synthase (GS), the GS-GOGAT pathway plays an important role in the assimilation of $\mathrm{NH}_{4}{ }^{+}$and de novo synthesis of glutamate in bacteria. Interestingly, whereas on plants inoculated with other ORS278 $\mathrm{Ndv}^{-}$mutants, small onsets of nodule organogenesis (i.e. bumps at the base of lateral roots) were detected, these observations were very rare or completely absent on plants inoculated with the ORS278 gltB::Tn5 and $g l t D:: \operatorname{Tn} 5$ mutant strains. These results are quite surprising as in 


\section{A glutamine +2 -oxoglutarate $\stackrel{\text { GOGAT }}{\longrightarrow}$ glutamate \\ $\mathrm{NH}_{4}^{+}+$2-oxoglutarate $\stackrel{\mathrm{GdhA}}{\rightleftarrows}$ glutamate}
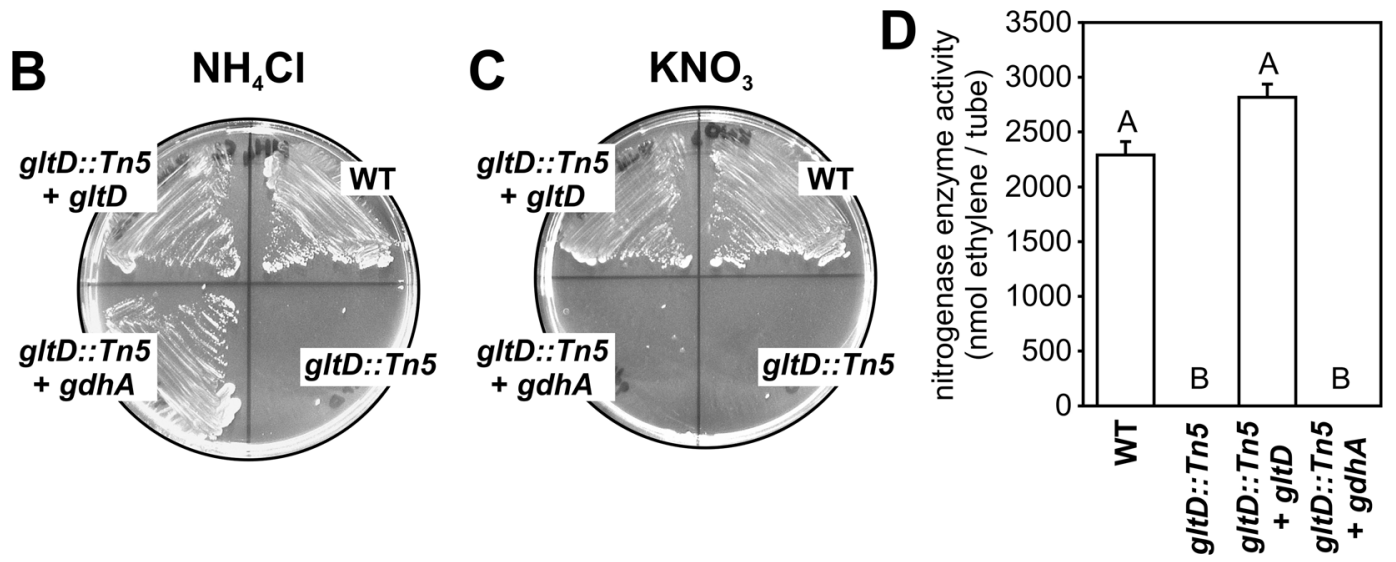

Figure 1. Growth and nitrogenase enzyme activity in free-living conditions of the Bradyrhizobium ORS285 gltD::Tn5 mutant. (A) Biosynthesis of glutamate catalysed by the glutamate synthase (GOGAT) and glutamate dehydrogenase (GdhA) enzyme. Growth of Bradyrhizobium ORS285 and derivatives on Minimal medium agar plates containing (B) $10 \mathrm{mM} \mathrm{NH}_{4} \mathrm{Cl}$ and (C) $10 \mathrm{mM} \mathrm{KNO}_{3}$ as nitrogen source. (D) ORS285 gltD::Tn5 mutant has no nitrogenase enzyme activity under free-living conditions. Ethylene production of ORS285 and derivatives grown for 5 days in vacuette tubes containing BNM-B agar $(0.8 \% \mathrm{w} / \mathrm{v})$ medium without nitrogen source and $10 \%$ acetylene gas. The mean amount of produced ethylene per tube $(n=4)$ is indicated. Error bars represent standard errors of the mean and letters represent conditions with significant difference according to the Tukey's test $(\mathrm{P}<0.05)$.

other rhizobium-legume interactions that depend on NFs, $g l t B$ and $g l t D$ mutants have no symbiotic defect ${ }^{5,6}$ or induce nodules that do not fix nitrogen (fix phenotype $)^{7-10}$. To investigate this divergence, we have characterized the growth characteristics and symbiotic phenotype of a Bradyrhizobium ORS285 gltD::Tn 5 mutant strain with both NF-independent and NF-dependent Aeschynomene species. The results show that the absence of GOGAT activity has a drastic effect on the growth on different nitrogen sources, and that in contrast to the interaction with NF-independent Aeschynomene species, the ORS285 gltD::Tn5 mutant strain is capable to induce nodules on NF-dependent host plants.

\section{Results}

Isolation and characterisation of a Bradyrhizobium ORS285 gltD::Tn5 mutant and construction of strains restoring ammonium assimilation. A Bradyrhizobium ORS285 gltD::Tn5 mutant was obtained by screening a random Tn5 insertion library of strain ORS285 for mutations that affect the symbiotic interaction with Aeschynomene indica and/or Aeschynomene afraspera, respectively. In one mutant strain with a symbiotic phenotype, the Tn5 insertion was found inside the gltD gene (BRAD285_V2_6186; Fig. 3A). The ORS285 gltD::Tn5 mutant strain was unable to grow on minimal medium plates containing ammonium $\left(\mathrm{NH}_{4}^{+}\right)$ as sole nitrogen source. The growth of this strain in medium containing $\mathrm{NH}_{4}{ }^{+}$could be restored when the WT gltD gene was re-introduced (strain ORS285 gltD::Tn $5+$ gltD) or by introducing the gdhA gene of E. coli MG1655 (strain ORS285 gltD::Tn5 + gdhA) (Fig. 1B). GdhA, encodes for a glutamate dehydrogenase, that is able to combine $\mathrm{NH}_{4}{ }^{+}$and 2-oxoglutarate to form 1 molecule of glutamate (Fig. 1A). These results indicate that: (1) the Tn5 insertion into the gltD gene is responsible for the inability to use $\mathrm{NH}_{4}{ }^{+}$as nitrogen source and (2) that the gene annotated as $g d h A$ in ORS285 (BRAD285_V2_4857) is not transcribed or the gene product is not able to assimilate $\mathrm{NH}_{4}^{+}$.

To analyse the effect of $g l t D$ inactivation on the usage of other nitrogen sources than $\mathrm{NH}_{4}{ }^{+}$, we have grown the ORS285 gltD::Tn5 mutant on succinate minimal BNM-B medium plates containing different amino acids and other compounds as $\mathrm{N}$-source and compared these to the growth of the WT strain, the ORS285 gltD::Tn $5+$ gltD strain and the ORS285 gltD::Tn5 + gdhA strain. Among the many compounds tested, the ORS285 gltD::Tn5 mutant was only able to grow on plates containing the amino acids asparagine, aspartic acid, glutamic acid, glutamine, leucine and isoleucine as $\mathrm{N}$-source (Table S1). To examine the utilization of these amino acids as $\mathrm{N}$-source in more detail, we have also investigated the growth in liquid minimal medium. This showed that in medium containing aspartic acid, glutamic acid, isoleucine, and leucine as N-source, the ORS285 gltD::Tn5 mutant strain grows faster and reaches a higher final optical density as compared to the WT strain (Fig. 2A). In 

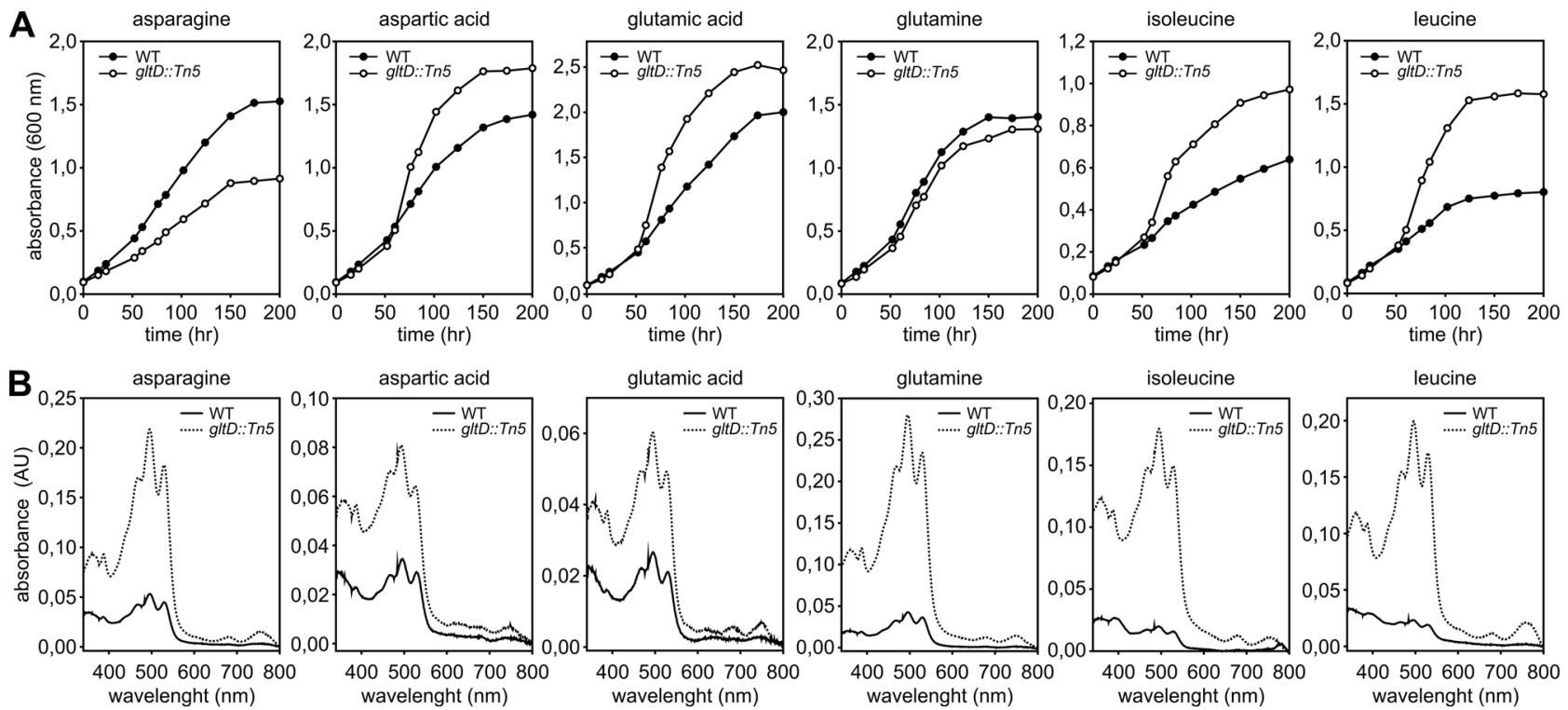

Figure 2. Growth and carotenoid production of the Bradyrhizobium ORS285 gltD::Tn5 strain using different amino acids as nitrogen source. (A) Representative growth curves of Bradyrhizobium ORS285 and Bradyrhizobium ORS285 gltD::Tn5 in minimal BNM-B medium containing different amino acids as nitrogen source. (B) Absorbance spectra of carotenoids extracted from $45 \mathrm{mg}$ (wet weight) Bradyrhizobium ORS285 and Bradyrhizobium ORS285 gltD::Tn5 cells at the end of the growth experiment shown in (A).

addition, analysing the carotenoid content of bacterial cells showed that the ORS285 gltD::Tn5 mutant strain on all tested amino acids, and in particular when using asparagine, glutamine, isoleucine and leucine as $\mathrm{N}$-source, produced a drastically increased amount of spirilloxanthin as compared to the WT strain suggesting that the photosynthetic activity is boosted in the mutant. (Fig. 2B). These experiments show that besides the inability to use certain $\mathrm{N}$-sources, the nitrogen and most probably carbon metabolism of the ORS285 gltD::Tn 5 mutant strain is drastically different from the WT strain. In the experiments, also a remarkable observation was made with the mutant strain expressing E. coli GdhA. The presence of the E. coli gdhA gene enabled the gltD::Tn5 mutant strain to use almost the same N-sources as WT ORS285 (Table S1). The only exceptions were the growth on $\mathrm{KNO}_{3}$ (Fig. 1C; Table S1) and reduction of atmospheric dinitrogen $\left(\mathrm{N}_{2}\right)$ as measured by the acetylene reduction assay (ARA) (Fig. 1D). This suggests that with these two $\mathrm{N}$-sources, the concentration of intracellular $\mathrm{NH}_{4}{ }^{+}$(and/or 2 -oxoglutarate) is not sufficient to form glutamate in the reaction catalysed by E. coli GdhA.

Expression of $g l t B / D$ operon in the presence of different $\mathrm{N}$-sources and in symbiosis. To measure $g l t B / D$ expression, we have constructed a reporter strain in which $g u s A$ gene was placed under the control of the promoter region of the $g l t B / D$ operon (Fig. $3 \mathrm{~A}$ ). This reporter strain (ORS285 $\mathrm{P}_{\mathrm{GOGAT}^{-}}$gusA) was subsequently grown in the presence of different nitrogen salts $\left(\mathrm{NH}_{4}{ }^{+} / \mathrm{NO}_{3}{ }^{-}\right)$or amino acids that allow growth of the ORS285 gltD::Tn 5 mutant strain and the $\beta$-glucuronidase activity was measured after $48 \mathrm{~h}$ of incubation at $28{ }^{\circ} \mathrm{C}$. Between the majority of analysed $\mathrm{N}$-sources there is only a slight (but statistical relevant) difference in $\beta$-glucuronidase activity (Fig. 3B). An exception are cells grown in the presence of the amino acid leucine for which the measured $\beta$-glucuronidase activity was 2 to 3.5 -fold higher as compared to cells grown in the presence of other $\mathrm{N}$-sources (Fig. 3B).

To investigate the expression of the $g l t B / D$ operon during symbiosis, the ORS285 $\mathrm{P}_{\mathrm{GOGAT}}-g u s A$ strain was used to infect the NF-independent and NF-dependent Aeschynomene species A. evenia and A. afraspera, respectively. Fifteen days post infection, some nodules were sliced and nodule sections were incubated with the $\beta$-glucuronidase substrate 5-bromo-4-chloro-3-indolyl-beta-D-glucuronic acid (X-Gluc). Blue staining of the central nodule tissue showed that the $g l t B / D$ operon is expressed in mature nodules of both $A$. evenia and A. afraspera plants (Fig. 3C, D). Although X-gluc staining is a qualitative measurement of gene expression, it should be noted that no significant difference in the colour could be detected by eye between the two species.

Glutamate synthase (GOGAT) activity is required for nodule organogenesis in NF-independent Aeschynomene evenia plants. Bradyrhizobium ORS285 has a relative broad host range and dependent on the plant species uses a NF-independent or NF-dependent mechanism to establish a symbiotic interaction with Aeschynomene legumes ${ }^{3}$. To investigate the effect of the GOGAT mutation in the symbiotic interaction with a NF-independent host plant, we infected our model species Aeschynomene evenia with the WT, and ORS285 gltD::Tn5, ORS285 gltD::Tn5 + gltD and ORS285 gltD::Tn5 + gdhA mutants. As previously observed for a GOGAT mutant in ORS278 on $A$. indica, the ORS285 gltD::Tn5 mutant failed to induce nodules on $A$. evenia. The most unexpected observation is the fact that the complementation of this mutant with gltD or $g d h A$ resulted in two completely distinct phenotypes. Whereas introducing the gltD gene into the ORS285 gltD::Tn5 mutant 

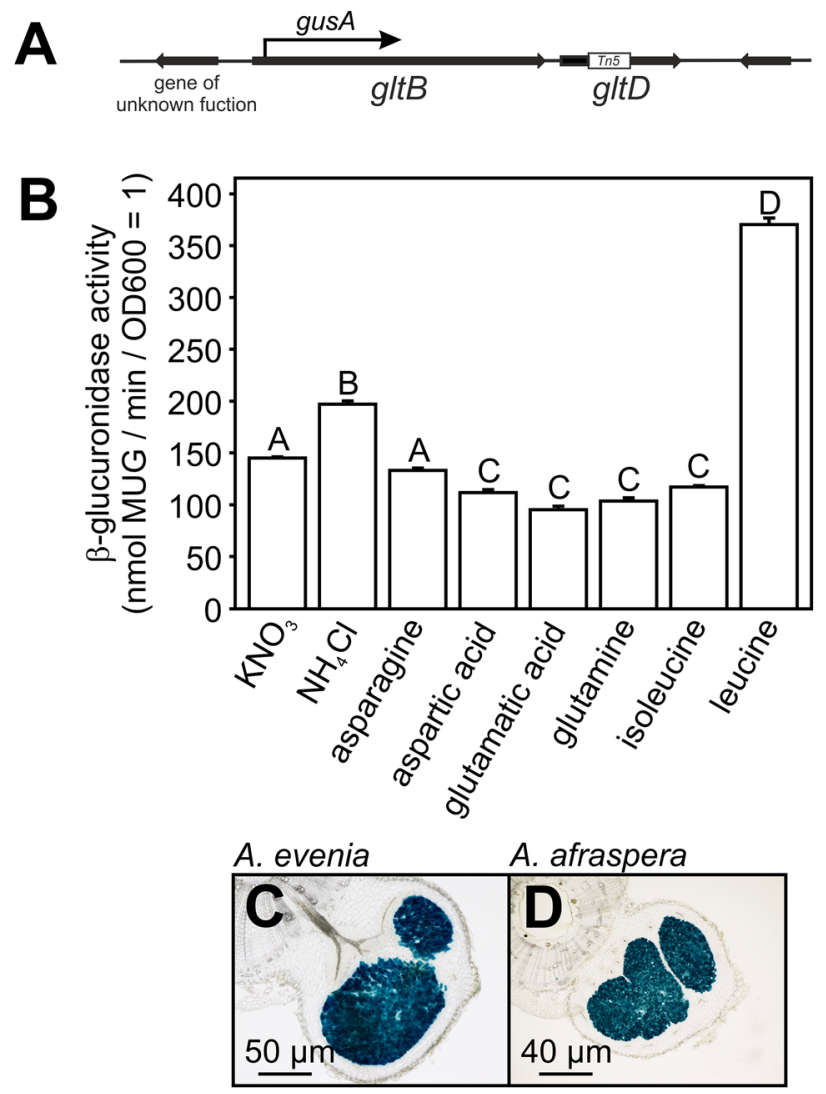

Figure 3. Activity of the Bradyrhizobium ORS285 GOGAT promoter under free-living and symbiotic conditions. (A) Schematic representation of the genomic region containing the gltB-gltD operon in Bradyrhizobium ORS285. The insertion of the Tn 5 transposon in the gltD gene is indicated by a white block and the point of the transcriptional fusion with the gusA gene in the $\mathrm{P}_{\mathrm{GOGAT}}$ reporter strain with and arrow. (B) $\beta$-glucuronidase activity of Bradyrhizobium ORS285 $\mathrm{P}_{\text {GOGAT }}$-gusA cells grown for $48 \mathrm{~h}$ in minimal BNM-B medium using the indicated nitrogen source. The results are from one representative experiment with three technical replicates for each experimental condition. Error bars represent standard errors of the mean and letters represent conditions with significant difference according to the Tukey's test $(\mathrm{P}<0.05)$. GOGAT promoter activity in nodules of (C) NF-independent $A$. evenia and (D) NF-dependent $A$. afraspera plants. A. evenia and $A$. afraspera plants were infected with the Bradyrhizobium ORS285 $\mathrm{P}_{\mathrm{GOGAT}}-g u s A$ reporter strain and at $15 \mathrm{dpi}$, the $\beta$-glucuronidase activity in $70 \mu \mathrm{M}$ nodule sections was determined by X-Gluc staining.

completely restored the plant growth, the plants inoculated with the ORS285 gltD::Tn $5+g d h A$ mutant showed reduced growth and a mild form of foliar chlorosis (Fig. 4A). Analysis of the roots showed that plants inoculated with the ORS285 gltD::Tn5 mutant did not contain nodules, whereas the roots of plants inoculated with the ORS285 gltD::Tn5 + gdhA mutant contained WT-like nodules (pink/rose colour) but the number was approximately half as compared to plants inoculated with the WT strain (Fig. 4B, C). In line with the visual observations, the acetylene reduction assay (ARA) showed that plants infected with the ORS285 gltD::Tn5 mutant had no nitrogenase enzyme activity, and those infected with ORS285 gltD::Tn5 + gdhA mutant an activity that was approximately half as observed with plants infected with the WT strain (Fig. 4D). Observations of the roots during the experiments showed that on plants inoculated with the ORS285 gltD::Tn5+gdhA mutant the first fullsized nodules appeared at 11-14 dpi whereas on plants inoculated with the WT strain this was at 3-5 dpi. As the ORS285 gltD::Tn5 + gdhA mutant had no nitrogenase enzyme activity under free-living conditions (Fig. 1D), we analysed if the formed nodules on ORS285 gltD::Tn5 $+g d h A$ inoculated plants were not due to a reversion of the gltD::Tn 5 mutation. Bacteria isolated from nodules at 23 dpi were kanamycin resistant and able to grow on minimal medium plates containing $\mathrm{NH}_{4} \mathrm{Cl}$ as $\mathrm{N}$-source (Fig. S1A, D). However in contrast to the original ORS285 gltD::Tn5 + gdhA mutant strain, bacteria re-isolated from the nodules were able to grow on plates containing $\mathrm{KNO}_{3}$ as nitrogen source (Fig. S1C). These results indicate that the observed nodules and nitrogenase enzyme activity on plants inoculated with the gltD::Tn5 + gdhA mutant are likely the result of a reversion of the gltD mutation. Plants inoculated with the mutant in which the WT gltD gene was re-introduced (ORS285 gltD::Tn5 + gltD ) were in all analysed characteristics indistinguishable from plants inoculated with the WT strain (Fig. 4A-D).

Growth experiments with the ORS285 gltD::Tn5 mutant showed that it can use only a few amino acids as nitrogen source (Table S1; Fig. 2A). To investigate if these amino acids can complement the symbiotic deficiency of the ORS285 gltD::Tn5 mutant, A. evenia plants were inoculated with the ORS285 gltD::Tn5 mutant strain in 
A

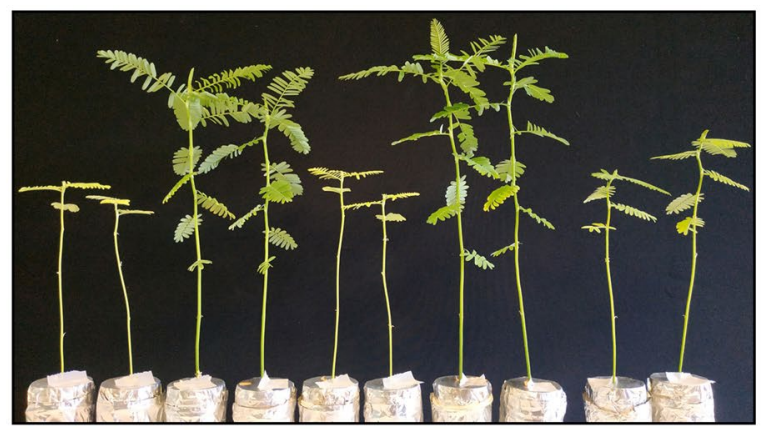

B

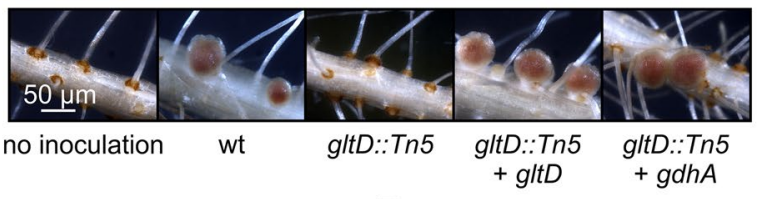

C
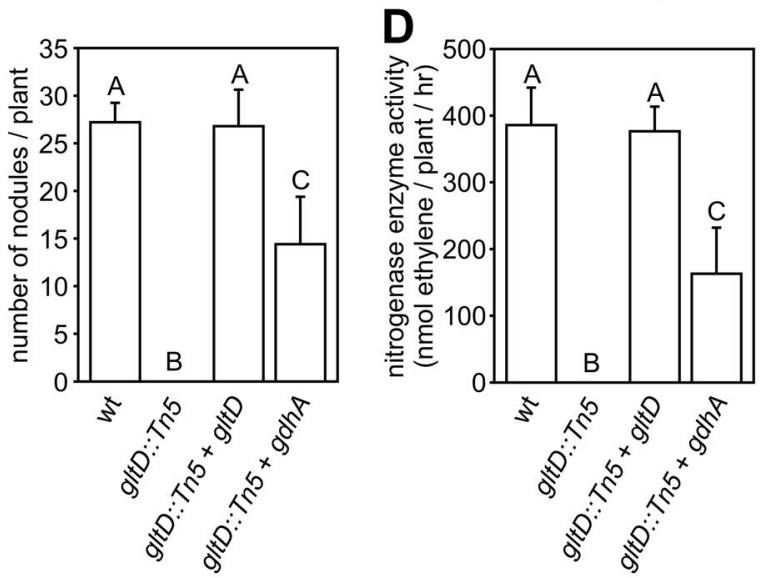

Figure 4. The Bradyrhizobium ORS285 gltD::Tn5 mutant does not induce nodules on Aeschynomene evenia (CIAT22838) plants. (A) Comparison of the growth of A. evenia plants inoculated with ORS285, ORS285 gltD::Tn5, ORS285 gltD::Tn5+gltD, and ORS285 gltD::Tn5 + gdhA. Non-inoculated plants (ni) were used as control. (B) Roots of A. evenia plants inoculated with ORS285, ORS285 gltD::Tn5, ORS285 gltD::Tn5 + gltD, and ORS285 gltD::Tn5 $+g d h A$. Note the absence of nodules on A. evenia plants inoculated with the ORS285 gltD::Tn5 strain. (C) Number of root nodules on A. evenia plants inoculated with ORS285, ORS285 gltD::Tn5, ORS285 gltD::Tn5 + gltD, and ORS285 gltD::Tn5 + gdhA, respectively. The mean number of nodules per plant $(\mathrm{n}=5)$ at 21 dpi is presented. (D) Acetylene reducing activity of $A$. evenia plants inoculated with ORS285, ORS285 gltD::Tn5, ORS285 gltD::Tn5 + gltD, and ORS285 gltD::Tn5 $+g d h A$ at 21 dpi. The mean amount of produced ethylene per hour and per plant $(n=5)$ is indicated. In $(\mathbf{C})$ and $(\mathbf{D})$ error bars represent standard errors of the mean and letters represent conditions with significant difference according to the Tukey's test $(\mathrm{P}<0.05)$.

the presence of $0.5 \mathrm{mM}$ of these different amino acids added to the plant growth medium. The addition of all these amino acids did not complement the symbiotic phenotype of the ORS285 gltD::Tn5 mutant. Nevertheless, in the case of glutamine, isoleucine or leucine addition, some of the A. evenia plants contained bump-like structures at the base of lateral roots whereas such structures were not observed when asparagine, aspartic acid or glutamic acid were added (Fig. 5A, B).

When examining the roots of ORS285 gltD::Tn5 infected plants, we noticed that the auxiliary root hairs at the base of a lot of lateral roots, which correspond to the site of infection, had a more intense brown colour as compared to the ones present on plants inoculated with the WT strain (Fig. 6A, C vs B, D). This is indicative for the accumulation of polyphenol compounds generally associated with plant defence reactions, as previously described in other legumes ${ }^{11}$.

NF-independent Aeschynomene species form a monophyletic clade that can be divided into four sub-groups ${ }^{12}$. To investigate whether the symbiotic requirement for GOGAT activity as found for A. evenia is a generality within this clade, we infected one species of the three other sub-groups (A. sensitiva, A. deamii, A. tambacoudensis) with the gltD::Tn5 mutant strain. With all analysed species, plants infected with the gltD::Tn5 mutant strain displayed: (1) nitrogen starvation symptoms (Fig. S2-S4, A); (2) the absence of nodules on their roots (Fig. S2-S4 B, C); (3) no nitrogenase enzyme activity (Fig. S2-S4, D). These data indicate that an active glutamate synthase complex (GOGAT) is obligatory for the induction of nodules on roots of NF-independent Aeschynomene species. 


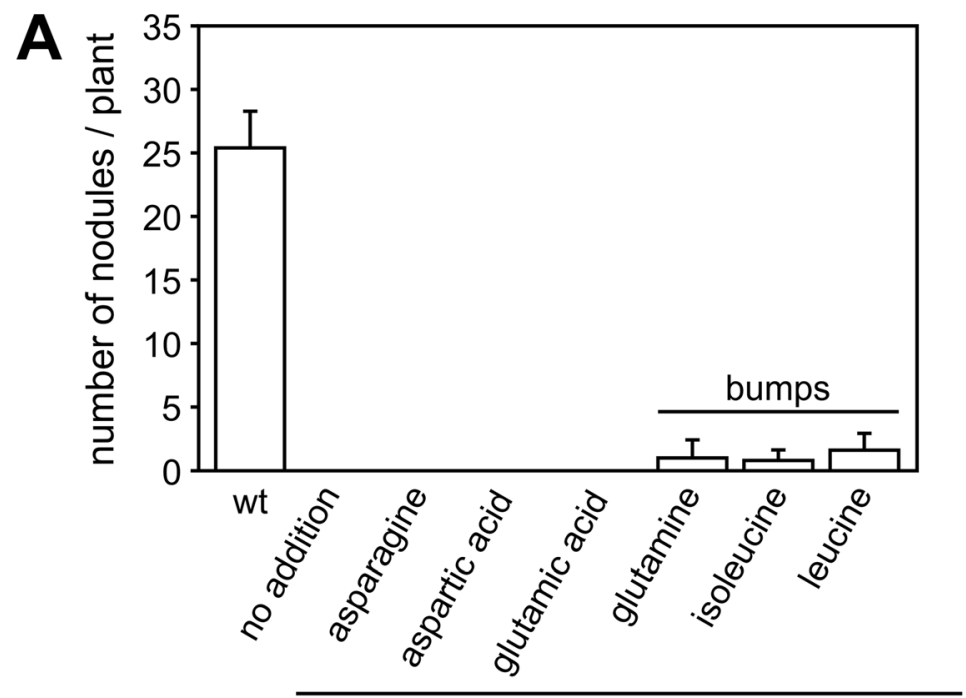

ORS285 gltD::Tn5

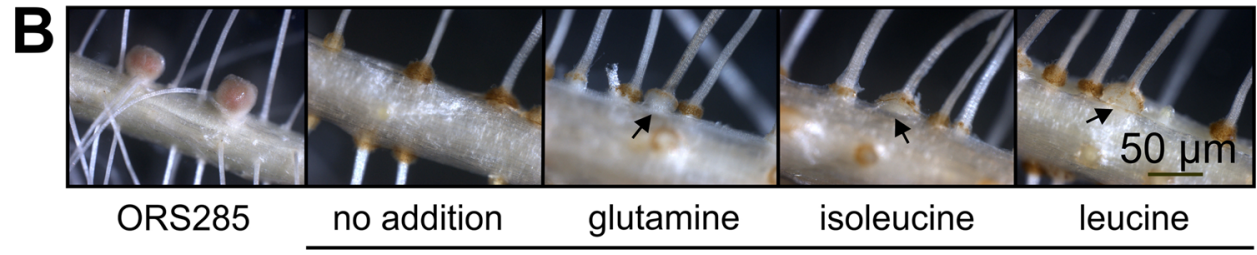

ORS285 gltD::Tn5

Figure 5. Amino acid supplementation does not restore the symbiotic phenotype of the Bradyrhizobium gltD::Tn5 mutant with A. evenia plants. (A) A. evenia plants were inoculated with the Bradyrhizobium ORS285 gltD::Tn 5 mutant in the presence of $0.5 \mathrm{mM}$ of the indicated amino acid and at $21 \mathrm{dpi}$ the number of nodules (cq. bumps) was determined. (B) mature nodules induced by wild-type ORS285 and small bumps induced by the ORS285 gltD::Tn5 mutant strain in the presence of $0.5 \mathrm{mM}$ glutamine, isoleucine or leucine on $A$. evenia roots.
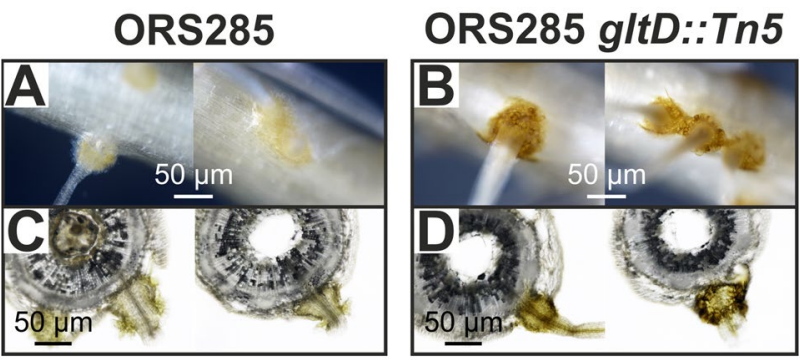

Figure 6. More intense browning of auxiliary root hairs on A. evenia plants inoculated with the Bradyrhizobium gltD::Tn5 mutant. Auxiliary root hairs of A. evenia plants inoculated with the (A) wild-type and (B) Bradyrhizobium gltD::Tn5 mutant strain at 5 dpi. $200 \mu \mathrm{M}$ coupes of root segments at 5 dpi to visualize the auxiliary root hairs of A. evenia plants inoculated with the (C) wild-type and (D) Bradyrhizobium gltD::Tn5 mutant strain.

In NF-dependent Aeschynome species the absence of glutamate synthase (GOGAT) activity results in a Fix phenotype. We also investigated the role of GOGAT activity in the symbiotic interaction with a NF-dependent host plant. For this, we infected the model species $A$. afraspera with the different mutant strains and observed the effect on plant growth, nodule number and nitrogenase enzyme activity at 20 dpi. Noninoculated, ORS285 gltD::Tn5 and ORS285 gltD::Tn5 $+g d h A$ infected plants had clear nitrogen starvation signs such as reduced plant growth and foliar chlorosis (Fig. 7A). In contrast to what observed with NF-independent Aeschynomene species, the ORS285 gltD::Tn5 mutant strain induced nodules on the roots of A. afraspera plants (Fig. 7B). However, the number of nodules on ORS285 gltD::Tn5 inoculated plants was about half the number 

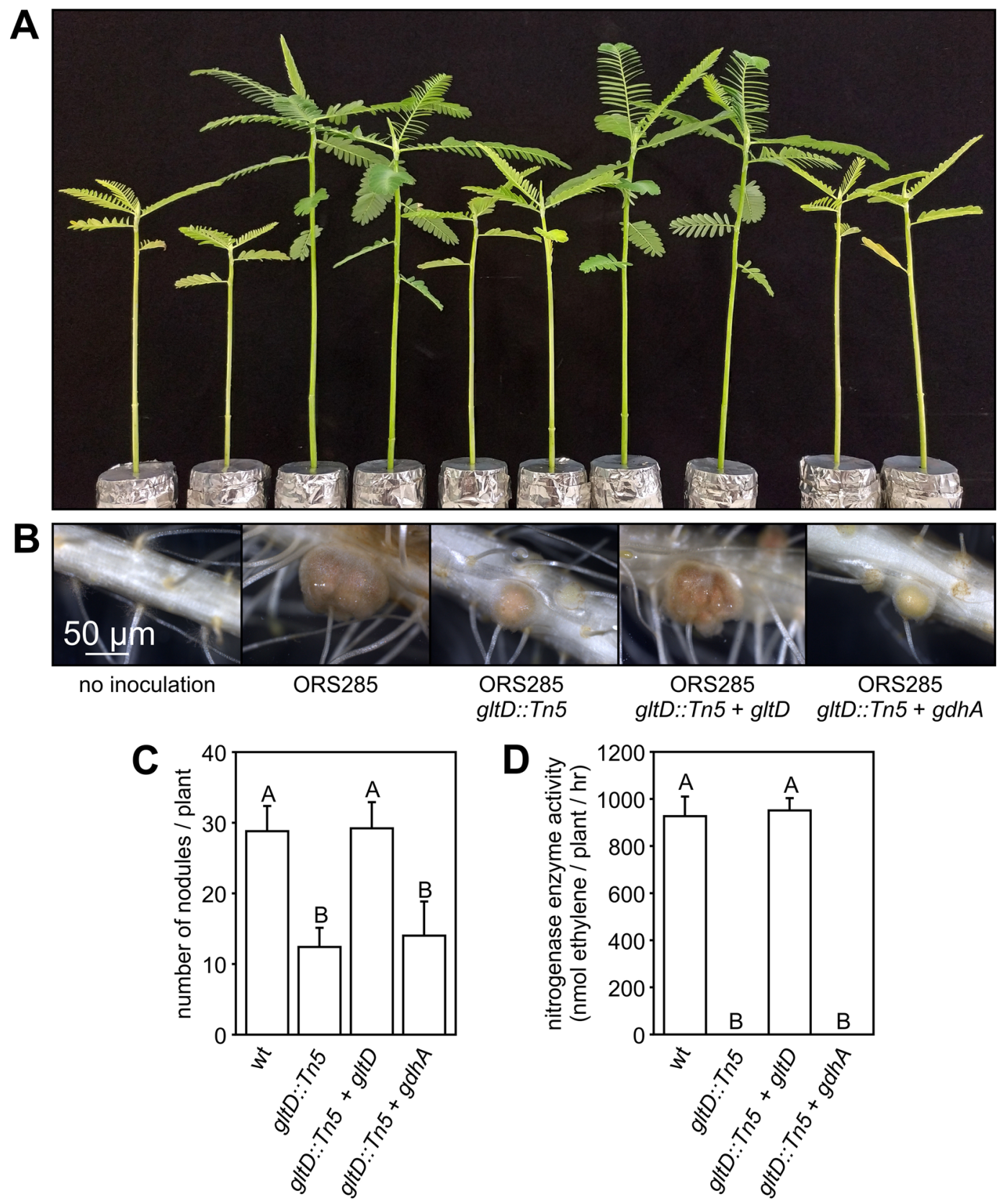

Figure 7. The Bradyrhizobium ORS285 gltD::Tn5 mutant does induce nodules on Aeschynomene afraspera (LSTM \#1) plants. (A) Comparison of the growth of A. afraspera plants inoculated with ORS285, ORS285 gltD::Tn5, ORS285 gltD::Tn5 + gltD, and ORS285 gltD::Tn5 + gdhA. Non-inoculated plants (ni) were used as control. (B) Roots of A. afraspera plants inoculated with ORS285, ORS285 gltD::Th5, ORS285 gltD::Tn5+gltD, and ORS285 gltD::Tn5 + gdhA. (C) Number of root nodules on A. afraspera plants inoculated with ORS285, ORS285 gltD::Tn5, ORS285 gltD::Tn5 + gltD, and ORS285 gltD::Tn5 + gdhA, respectively. The mean number of nodules per plant $(\mathrm{n}=5)$ at $21 \mathrm{dpi}$ is presented. (D) Acetylene reducing activity of $A$. afraspera plants inoculated with ORS285, ORS285 gltD::Tn5, ORS285 gltD::Tn5 + gltD, and ORS285 gltD::Tn5+gdhA at 21 dpi. The mean amount of produced ethylene per hour and per plant $(n=5)$ is indicated. In $(\mathbf{C})$ and (D) error bars represent standard errors of the mean and letters represent conditions with significant difference according to the Tukey's test $(\mathrm{P}<0.05)$.

as compared to WT inoculated plants and their colour was light-pink/white in contrast to red/pink as found for WT nodules (Fig. 7B, C). In line with these observations, no nitrogenase enzyme activity was detected in the ARA assay on plants inoculated with the ORS285 gltD::Tn5 mutant (Fig. 7D). Exactly the same observations 

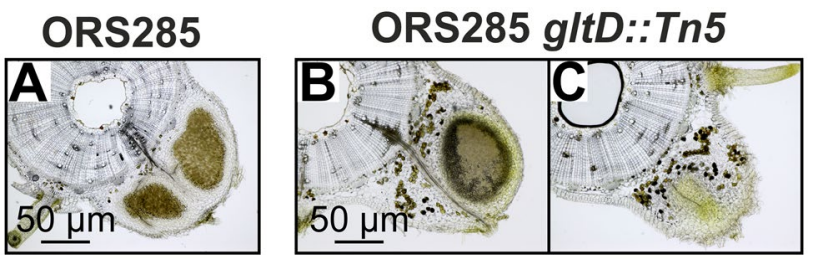

Figure 8. A. afraspera nodules induced by the Bradyrhizobium ORS285 gltD::Tn5 mutant contain many plant cells with a gold-brown substance. $70 \mu \mathrm{M}$ nodule sections of $A$. afraspera plants inoculated with (A) wild-type ORS285 and (B), (C) ORS285 gltD::Tn5 mutant. Note the abundant presence of plant cells containing a goldbrown substance in nodule sections of plants inoculated with the ORS285 gltD::Tn5 mutant strain.

were made with plants that had been inoculated with the ORS285 gltD::Tn $5+g d h A$ mutant, whereas the ORS285 gltD::Tn $5+$ gltD mutant behaved like the WT strain.

Cytological analysis of nodules induced by ORS285 gltD::Tn5 strain, showed that the bacterial infection of nodules induced by the ORS285 gltD::Tn 5 mutant strain was relatively heterogeneous (Fig. 8B, C). Some nodules contained a large infected central tissue like as found in WT nodules (Fig. 8, A vs B), whereas in other nodules this "zone" was absent or small (Fig. 8C). Moreover, in sections of the ORS285 gltD::Tn5 nodules, and in particular in the ones without a central infected zone, numerous plant cells having a typical brown colour were observed. This suggests that the infection by the ORS285 gltD::Tn 5 mutant strain induced also defence responses in $A$. afraspera plants but probably to a less extent than observed in A. evenia.

We also tested the GOGAT mutant with another NF-dependent Aeschynomene species, A. nilotica. With this plant species, the gltD::Tn5 mutant had exactly the same symbiotic phenotype, i.e. nitrogen starvation symptoms, reduced nodule number and no nitrogenase enzyme activity, as observed with A. afraspera plants (Fig. S5, A-D). In addition, a light brown colour at the outer surface of the nodules and presence of plant cells with an intense brown colour inside the nodule tissue were observed on plants infected with the ORS285 gltD::Tn5 strain (Fig. S5, E vs. F, G). Thus upon interaction with members of the group of NF-dependent Aeschynomene species, the ORS285 gltD::Tn5 mutant is able to induce nodule organogenesis and to infect the plant tissue but this interaction does not lead to a nitrogen fixing symbiosis.

\section{Discussion}

In a previous Tn5 mutagenesis study to identify genes important for the NF-independent symbiotic interaction of photosynthetic Bradyrhizobium ORS278 with Aeschynomene indica, we observed that strains with Tn5 insertion into the gltB or gltD gene had the strongest $\mathrm{ndv}^{-}$(nodule development) phenotype ${ }^{4}$. In this report, we have characterized a glutamate synthase (GOGAT; gltD::Tn5) mutant of Bradyrhizobium ORS285 and its symbiotic properties with NF-dependent and NF-independent Aeschynomene species. As shown for GOGAT mutants of other rhizobia ${ }^{5-7,9,10}$, the ORS285 gltD::Tn5 mutant is unable to use ammonium, nitrate and several amino acids as nitrogen source (Table S1). In bacteria, ammonium assimilation can take place via the glutamine synthase (GS) -glutamate synthase (GOGAT) pathway or via the enzyme glutamate dehydrogenase (GdhA) (Fig. 1A). Genome analysis showed that the ORS285 strain, in contrast to many other rhizobia, has a glutamate dehydrogenase (GDH) homologue (BRAD285_V2_4857) which displays 48\% of identity with the GDH of the hyperthermophilic bacterium Thermotoga maritima. However, despite the presence of this gdh gene and transcription under free-living conditions, the gltD::Tn5 mutant strain is incapable to use $\mathrm{NH}_{4}^{+}$as nitrogen source. Interestingly, when the $E$. coli gdhA gene was introduced into the ORS285 gltD::Tn5 mutant, the strain was capable to grow on $\mathrm{NH}_{4}{ }^{+}$and amino acids as found for the wild-type strain (Fig. 1B; Table S1). This indicates that the $\mathrm{NH}_{4}{ }^{+}$and 2-oxoglutarate concentrations in the ORS285 cell are sufficient high for ammonium assimilation via the GDH pathway but that the BRAD285_V2_4857 gene product is unable to do so and probably only has glutamate dehydrogenase activity. Surprisingly, the E. coli gdhA gene was unable to restore growth of the gltD::Tn5 mutant on $\mathrm{NO}_{3}{ }^{-}$plates (Fig. 1C) and no nitrogenase enzyme activity was detected in the acetylene reduction (ARA) assay under free-living conditions (Fig. 1D). Enzymes involved in nitrate and atmospheric nitrogen reduction are induced and have a complex regulation mechanism that also involve glutamine and 2-oxoglutarate concentrations in the cell ${ }^{13,14}$. We hypothesize that in the absence of GOGAT activity, the metabolism and glutamine/2-oxoglutarate concentrations in the cell are drastically altered and that this causes that the ORS285 gltD::Tn5 mutant is incapable to induce the nif and nitrate reductase genes despite the presence of E. coli gdhA. In line with an altered metabolism in the ORS285 gltD::Tn 5 mutant, is the observation that this strain grows faster and produces drastically more photosynthetic pigment as compared to the wild-type ORS285 strain when using certain amino acids as nitrogen source (Fig. 2A, B). Thus besides the inability of the gltD::Tn5 mutant to assimilate ammonium and to synthesize glutamate via the GS-GOGAT pathway, the regulation of nitrogen and carbon metabolism in the mutant bacteria seems to be largely altered.

In this study, we show that GOGAT activity in ORS285 is absolutely required for nodule organogenesis in the interaction with NF-independent Aeschynomene species. In other rhizobium-legume interactions, different symbiotic phenotypes of GOGAT mutants have been described, but none has been that drastic as observed for the gltD and gltB/D mutants of ORS285 (this study) and ORS278 ${ }^{4}$, respectively, upon interaction with NFindependent Aeschynomene legumes. Whereas GOGAT mutants of Bradyrhizobium diazoefficiens and Azorhizobium caulinodans are forming nodules that are unable to fix nitrogen (O’Gara et al., 1984; Hilgert et al., 1987), a 
Sinorhizobium meliloti mutant does not have a symbiotic phenotype. Moreover, the absence of GOGAT activity in Sinorhizobium etli results in a more efficient symbiosis (Castillo et al., 2000). As all above described symbiotic interactions depend on NFs, the nodule organogenesis minus phenotype of the ORS285 gltD::Tn 5 mutant with certain Aeschynomene species could thus be related to the NF-independent character of the symbiotic interaction. In line with this hypothesis are the observations that the ORS285 gltD::Tn5 mutant is able to induce nodules on the NF-dependent Aeschynomene species A. afraspera and A. nilotica (Fig. 7; Fig. S5) However, the induced nodules on the NF-dependent Aeschynomene species are unable to fix atmospheric nitrogen (Fig. 7D; Fig. S5, D). The latter is not so surprising as the ORS285 gltD::Tn5 mutant also did not have nitrogenase enzyme activity under free-living conditions (Fig. 1D).

Growth experiments showed that the ORS285 gltD::Tn5 mutant can only use a limited number of amino acids as nitrogen source (Table S1). As these amino acids could be limiting in the plant environment, we did experiments were we added amino acids that can be used by the ORS285 gltD::Tn 5 mutant to the plant growth medium (0.5 mM final concentration) (Fig. 5). With some amino acids (glutamine, isoleucine, leucine) this resulted in the rare formation of very small bumps on some of the ORS285 gltD::Tn5 inoculated plants. We therefore believe that a reduced growth of the ORS285 gltD::Tn5 mutant is not the main cause of the nodule organogenesis minus phenotype as observed with NF-independent Aeschynomene species. Observing the auxiliary root hairs of A. evenia plants inoculated with the ORS285 gltD::Tn 5 mutant, we noticed at a few days post infection a more intense browning of the axillary root hairs as compared to plants inoculated with the wild-type ORS285 (Fig. 6). This suggests that plants inoculated with the ORS285 gltD::Tn5 mutant respond with an accumulation of polyphenols at the site of colonization and infection by the bacterium. Interestingly, an intense browning of multiple plant cells inside nodules was also observed on A. afraspera and A. nilotica plants inoculated with the ORS285 gltD::Tn5 mutant (Fig. 8B, C; Fig. S5, F, G). Moreover, whereas mutant rhizobia unable to fix atmospheric nitrogen normally induce more nodules on plants as compared to the wild-type strain, the ORS285 gltD::Tn5 mutant strain induces about half the amount of nodules on NF-dependent Aeschynomene species as compared to wild type ORS285 (Fig. 7C; Fig. S5, C). We therefore hypothesize that ORS285 gltD::Tn5 bacteria induce a plant immune response in Aeschynomene plants, which is sufficient to completely inhibit nodule organogenesis and infection in NF-independent Aeschynomene species, but which, likely due to the perception of NFs, is to some extent suppressed in NF-dependent Aeschynomene species.

At this stage it is difficult to point what in the ORS285 gltD::Tn5 mutant causes the immune response in Aeschynomene plants. The growth experiments showed that the (nitrogen) metabolism in ORS285 gltD::Tn5 mutant bacteria is drastically different than in wild-type bacteria. This may lead to different proteins and or molecules at the cell surface of ORS285 gltD::Tn5 mutant bacteria that are incompatible with a symbiotic interaction. Alternatively, a more restricted metabolism due to the absence of GOGAT enzyme activity in the ORS285 gltD::Tn5 mutant could lead that the bacteria are not capable to adapt to the symbiotic conditions leading to bacterial cell lysis and subsequent induction of a plant immune response.

\section{Material and methods}

Bacterial strains and growth conditions. Tables of strains and plasmids used in this study can be found in the Supporting information (Tables S2, S3). Bradyrhizobium ORS285 $5^{15}$ and derivatives were grown at $28^{\circ} \mathrm{C}$ in modified yeast extract mannitol medium $\left(\mathrm{YM}^{16}\right)$ or a minimal BNM-B medium ${ }^{17}$ with $10 \mathrm{mM}$ succinate as carbon source and $10 \mathrm{mM}$ ammonium chloride as nitrogen source, unless otherwise indicated. When required, ampicillin $(50-100 \mu \mathrm{g} / \mathrm{ml})$, kanamycin $(50-120 \mu \mathrm{g} / \mathrm{ml})$, or cefotaxime $(20 \mu \mathrm{g} / \mathrm{ml})$ were added to the growth medium.

Isolation of a Bradyrhizobium ORS285 gltD::Tn5 mutant. A Bradyrhizobium ORS285 Tn5 transposon library was constructed via a biparental mating protocol as described by ${ }^{18}$ using E. coli BW20767 containing plasmid pCRS487 harbouring the mini-transposon $\mathrm{mTn} 5-\mathrm{GNm}$ as donor strain ${ }^{19}$. Approximately five thousand kanamycine resistant clones were isolated from several independent matings and analysed for their symbiotic phenotype on $A$. indica and $A$. afraspera plants as described in Bonaldi et al. ${ }^{4}$. Mutants showing a symbiotic phenotype were retained and the Tn5 insertion site was determined. One of the isolated mutants had the mTn5$\mathrm{GNm}$ transposon inserted into the gltD gene.

Complementation of the ORS285 gltD::Tn5 mutant. Complementation of growth of the ORS285 gltD::Tn5 mutant on minimal medium plates containing ammonium chloride as nitrogen source was tested using two different approaches: 1) restoring the mutant gltD gene with a wild-type copy of the gene, 2) introduction of the glutamate dehydrogenase gene $(g d h A)$ of E. coli MG1655. The Bradyrhizobium ORS285 gltD and E. coli MG1655 gdhA DNA regions were PCR amplified using primers that can be found in Table S4 of the supplementary information and cloned into pGEM-T Easy (Promega) and transformed into thermocompetent E. coli XL2-Blue cells (Agilent). Correct clones were verified by sequence analysis. The fragment containing the complete ORS285 gltD gene in pGEM-T Easy was excised with SpeI-BamHI and ligated into suicide vector pJG194 ${ }^{20}$ digested with SpeI-BamHI. After transformation into E. coli XL2 Blue cells, correct clones were selected via kanamycin resistance $(50 \mu \mathrm{g} / \mathrm{ml})$ and subsequent DNA restriction enzyme analysis. The $E$. coli MG1655 gdhA gene in pGEM-T Easy was excised with BamHI-EcoRI and ligated into suicide vector pJG194-4694-miaA ${ }^{17}$ digested with BamHI-EcoRI. This places the E. coli MG1655 gdhA gene under control of a promoter that is constitutively expressed in Bradyrhizobium ORS285. pJG194 plasmids containing the ORS285 gltD and E. coli gdhA gene, respectively, were transformed into $\mathrm{CaCl}_{2}$ competent $E$. coli $\mathrm{S} 17-1$ cells ${ }^{21}$ and mobilized into the Bradyrhizobium ORS285 gltD::Tn5 mutant using the biparental mating protocol as previously described ${ }^{17}$. After mating, ORS285 $g l t D:: \operatorname{Tn} 5$ bacteria in which the plasmid has been integrated into the chromosome were selected on minimal 
medium plates containing $120 \mu \mathrm{g} / \mathrm{ml}$ kanamycine, $20 \mu \mathrm{g} / \mathrm{ml}$ cefotaxime and $10 \mathrm{mM}$ ammonium chloride as nitrogen source. Correct insertion of the plasmids was verified by PCR.

Construction of a GOGAT reporter strains of Bradyrhizobium ORS285. To construct a GOGAT reporter strain, the 576 bp region upstream of the $g l t B-g l t D$ was amplified by PCR using the primers indicated in Table S3 (Supplementary information), cloned into pGEM-T Easy and transformed into E. coli XL2-Blue cells. After verification by sequence analysis, the promoter fragment was excised with SalI-BamHI and ligated into suicide vector pJG194-PnodA-gus $A^{22}$ digested with the same enzymes. After transformation into E. coli XL2 Blue cells, correct clones were selected as described above. Plasmids were transformed into $\mathrm{CaCl}_{2}$ competent $E$. coli S17-1 cells ${ }^{21}$ and mobilized into Bradyrhizobium ORS285 using the biparental mating ${ }^{17}$. Correct insertion of the plasmids was verified by PCR.

Expression of the gltB-gltD operon in the presence of different $\mathbf{N}$-sources. The Bradyrhizobium ORS285 $\mathrm{P}_{\mathrm{GOGAT}}$-gusA reporter strain was used to measure the expression of the gltB-gltB operon when bacteria are grown in minimal BNM-B medium ${ }^{17}$ containing potassium nitrate, ammonium chloride or different amino acids (10 mM final concentration) as nitrogen source. Bradyrhizobium ORS285 $\mathrm{P}_{\mathrm{GOGAT}^{-}}$-gusA was grown $\mathrm{YM}$ medium for three days at $28{ }^{\circ} \mathrm{C}$. The bacteria were harvested, washed two times with BNM-B medium without carbon and nitrogen source and subsequently diluted into BNM-B medium (OD600 =0.1) containing $10 \mathrm{mM}$ succinate as carbon source and the indicated nitrogen source $(10 \mathrm{mM})$. After $48 \mathrm{~h}$ of growth at $28^{\circ} \mathrm{C}$, the absorbance at $600 \mathrm{~nm}$ of the cultures were determined and the $\beta$-glucuronidase activities were measured according to the method of Miller ${ }^{23}$ using 4-methylumbelliferyl- $\beta$-D-glucuronide (Sigma-Aldrich) as substrate. Experiments were at least repeated twice with three technical replicates in one experiment.

In vitro nitrogenase enzyme activity. To measure the nitrogenase enzyme activity under free-living conditions, Bradyrhizobium ORS285 and derivatives were grown in $2 \mathrm{ml} \mathrm{BNM-B} \mathrm{0.8 \%} \mathrm{agar} \mathrm{medium} \mathrm{in} 9 \mathrm{ml}$ vacuette tubes (Greiner bio-one; ref: 455001). To circumvent overpressure, $0.9 \mathrm{ml}$ of air was removed before injecting the same volume of $100 \%$ acetylene. The cultures were incubated at $28^{\circ} \mathrm{C}$ and at 5 days $1 \mathrm{ml}$ gas samples were analysed for ethylene production by gas chromatography ${ }^{24}$.

Carotenoid extraction. After 8 days of growth at $28{ }^{\circ} \mathrm{C}$ in minimal BNM-B medium containing different amino acids as nitrogen source, bacteria were harvested by centrifugation ( $15 \mathrm{~min} 4000 \times \mathrm{g})$. After complete removal of the culture supernatant, the weight of the cell pellet was determined, whereafter carotenoids were extracted by dissolving $45 \mathrm{mg}$ (wet weight) cells in a $1 \mathrm{ml}$ acetone/methanol (7:2) mixture. After $5 \mathrm{~h}$ incubation with regular mixing on ice, the mixture was centrifuged for $10 \mathrm{~min}$ at $20,000 \times \mathrm{g}$ in an Eppendorf centrifuge, whereafter the supernatant was collected. To determine the amount of carotenoids in the sample an absorbance scan (300-800 nm; $1 \mathrm{~nm}$ steps) of the supernatant was made using a VARIAN Cary 50 photo spectrometer.

Plant growth and acetylene reduction assay. In this study A. evenia (CIAT22838; Malawi), A. deamii (LSTM \#24; Mexico), A. tambacoudensis (LSTM \#60; Senegal), and A. sensitiva (LSTM \#28; Senegal) were used as model NF-independent Aeschynomene species and A. afraspera (LSTM \#1; Senegal), and A. nilotica (IRRI 014040; Senegal) as model NF-dependent ones. All seeds from above indicated Aeschynomene species were produced in greenhouses of our laboratory at the Laboratoire des Symbioses Tropicales et Méditerranéennes (LSTM), Montpellier, France. Sterilization of seeds, germination, growth of plants and inoculation with Bradyrhizobium ORS285 and derivatives were as described ${ }^{24}$. After 21 days post-inoculation, photos of plants were taken, the amount of nodules on the roots were counted and the acetylene reduction assay (ARA) was used to measure the nitrogenase enzyme activity ${ }^{17}$. In experiments to determine if supplementation of amino acids could restore the nodule organogenesis minus phenotype of the ORS285 gltD::Tn 5 mutant, amino acids (final concentration $0.5 \mathrm{mM}$ ) were added to the plant growth medium $1 \mathrm{~h}$ prior to bacterial inoculation. All plant experimental research was carried out in accordance with relevant institutional, national and international guidelines and legislation.

Microscopy. To determine the glucuronidase activity in nodules formed by the $\mathrm{P}_{\mathrm{GOGAT}^{-}}$-reporter strain, fresh nodules were embedded in $5 \%$ agarose and sectioned $(50-70 \mu \mathrm{M})$ using a vibratome (VT1000S; Leica, Nanterre). Nodule slices were incubated for $30 \mathrm{~min}$ in GUS assay buffer ${ }^{2}$ at $37^{\circ} \mathrm{C}$. After staining the slices were washed three times with water, mounted on microscope slides and observed under bright-field illumination with a macroscope (Nikon AZ100; Champigny-sur-Marne, France).

Received: 6 August 2021; Accepted: 11 October 2021

Published online: 22 October 2021

\section{References}

1. Giraud, E. \& Fleischman, D. Nitrogen-fixing symbiosis between photosynthetic bacteria and legumes. Photosynth. Res. 82, 115-130 (2004).

2. Bonaldi, K. et al. Nodulation of Aeschynomene afraspera and A. indica by photosynthetic Bradyrhizobium sp. strain ORS285: The nod-dependent versus the nod-independent symbiotic interaction. Mol. Plant. Microbe. Interact. 24, 1359-1371 (2011).

3. Giraud, E. et al. Legumes symbioses: Absence of nod genes in photosynthetic bradyrhizobia. Science 316, 1307-1312 (2007). 
4. Bonaldi, K. et al. Large-scale transposon mutagenesis of photosynthetic Bradyrhizobium sp. strain ORS278 reveals new genetic loci putatively important for nod-independent symbiosis with Aeschynomene indica. Mol. Plant-Microbe Interact. 23, 760-770 (2010).

5. Lewis, T. A., Gonzalez, R. \& Botsford, J. L. Rhizobium meliloti glutamate synthase: Cloning and initial characterization of the glt locus. J. Bacteriol. 172, 2413-2420 (1990).

6. Castillo, A. et al. Role of GOGAT in carbon and nitrogen partitioning in Rhizobium etli. Microbiology 146, 1627-1637 (2000).

7. O'Gara, F., Manian, S. \& Meade, J. Isolation of an Asm-mutant of Rhizobium japonicum defective in symbiotic N2-fixation. FEMS Microbiol. Lett. 24, 241-245 (1984).

8. Hilgert, U., Schell, J. \& de Bruijn, F. J. Isolation and characterization of Tn5-induced NADPH-glutamate synthase (GOGAT-) mutants of Azorhizobium sesbaniae ORS571 and cloning of the corresponding glt locus. Mol. Gen. Genet. MGG 210, 195-202 (1987).

9. Ferraioli, S. et al. Auxotrophic mutant strains of Rhizobium etli reveal new nodule development phenotypes. Mol. Plant-Microbe Interact. 15, 501-510 (2002).

10. Mulley, G. et al. Mutation of GOGAT prevents pea bacteroid formation and N2 fixation by globally downregulating transport of organic nitrogen sources. Mol. Microbiol. 80, 149-167 (2011).

11. Bourcy, M., Berrabah, F., Ratet, P. \& Gourion, B. Failure of self-control: Defense-like reactions during legume/rhizobia symbiosis. Plant Signal. Behav. 8, e23915 (2013).

12. Chaintreuil, C. et al. Naturally occurring variations in the nod-independent model legume Aeschynomene evenia and relatives: A resource for nodulation genetics. BMC Plant Biol. 18, 54 (2018).

13. Fischer, H. M. Genetic regulation of nitrogen fixation in rhizobia. Microbiol. Rev. 58, 352-386 (1994).

14. López, M. F., Cabrera, J. J., Salas, A., Delgado, M. J. \& López-García, S. L. Dissecting the role of NtrC and RpoN in the expression of assimilatory nitrate and nitrite reductases in Bradyrhizobium diazoefficiens. Antonie Van Leeuwenhoek 110, 531-542 (2017).

15. Molouba, F. et al. Photosynthetic bradyrhizobia from Aeschynomene spp. are specific to stem-nodulated species and form a separate 16S ribosomal DNA restriction fragment length polymorphism group. Appl. Environ. Microbiol. 65, 3084-3094 (1999).

16. Giraud, E., Hannibal, L., Fardoux, J., Verméglio, A. \& Dreyfus, B. Effect of Bradyrhizobium photosynthesis on stem nodulation of Aeschynomene sensitiva. Proc. Natl. Acad. Sci. U. S. A. 97, 14795-14800 (2000)

17. Podlešáková, K. et al. Rhizobial synthesized cytokinins contribute to but are not essential for the symbiotic interaction between photosynthetic bradyrhizobia and Aeschynomene legumes. Mol. Plant-Microbe Interact. 26, 1232-1238 (2013).

18. Jaubert, M., Hannibal, L., Fardoux, J., Giraud, E. \& Verméglio, A. Identification of novel genes putatively involved in the photosystem synthesis of Bradyrhizobium sp. ORS278. Photosynth. Res. 100, 97-105 (2009).

19. Reeve, W. G. et al. Constructs for insertional mutagenesis, transcriptional signal localization and gene regulation studies in root nodule and other bacteria. Microbiology 145, 1307-1316 (1999).

20. Griffitts, J. S. \& Long, S. R. A symbiotic mutant of Sinorhizobium meliloti reveals a novel genetic pathway involving succinoglycan biosynthetic functions. Mol. Microbiol. 67, 1292-1306 (2008).

21. Simon, R., Priefer, U. \& Pühler, A. A broad host range mobilization system for in vivo genetic engineering: Transposon mutagenesis in Gram negative bacteria. Bio/Technology 1, 784-791 (1983).

22. Nouwen, N., Arrighi, J. F., Gully, D. \& Giraud, E. RibBX of Bradyrhizobium ORS285 plays an important role in intracellular persistence in various Aeschynomene host plants. Mol. Plant-Microbe Interact. 34, 88-99 (2021).

23. Miller, J. H. Experiments in Molecular Genetics (CSH Laboratory Press, 1972).

24. Nouwen, N. et al. The role of rhizobial (NifV) and plant (FEN1) homocitrate synthases in Aeschynomene/photosynthetic Bradyrhizobium symbiosis. Sci. Rep. 7, 448 (2017)

25. Blattner, F. R. et al. The complete genome sequence of Escherichia coli K-12. Science 277, 1453-1462 (1997).

\section{Acknowledgements}

This study was supported by the ANR grants 'SymEffectors' (ANR-16-CE20-0013) and 'ET-Nod' (ANR-20-CE20-0012).

\section{Author contributions}

N.N. conceived and designed this study. N.N., C.C. and J.F. conducted the experiments. N.N. analyzed the data and prepared the manuscript assisted by E.G. All authors reviewed the manuscript.

\section{Competing interests}

The authors declare no competing interests.

\section{Additional information}

Supplementary Information The online version contains supplementary material available at https://doi.org/ 10.1038/s41598-021-00480-7.

Correspondence and requests for materials should be addressed to N.N.

Reprints and permissions information is available at www.nature.com/reprints.

Publisher's note Springer Nature remains neutral with regard to jurisdictional claims in published maps and institutional affiliations.

(c) (i)

Open Access This article is licensed under a Creative Commons Attribution 4.0 International License, which permits use, sharing, adaptation, distribution and reproduction in any medium or format, as long as you give appropriate credit to the original author(s) and the source, provide a link to the Creative Commons licence, and indicate if changes were made. The images or other third party material in this article are included in the article's Creative Commons licence, unless indicated otherwise in a credit line to the material. If material is not included in the article's Creative Commons licence and your intended use is not permitted by statutory regulation or exceeds the permitted use, you will need to obtain permission directly from the copyright holder. To view a copy of this licence, visit http://creativecommons.org/licenses/by/4.0/.

(c) The Author(s) 2021 